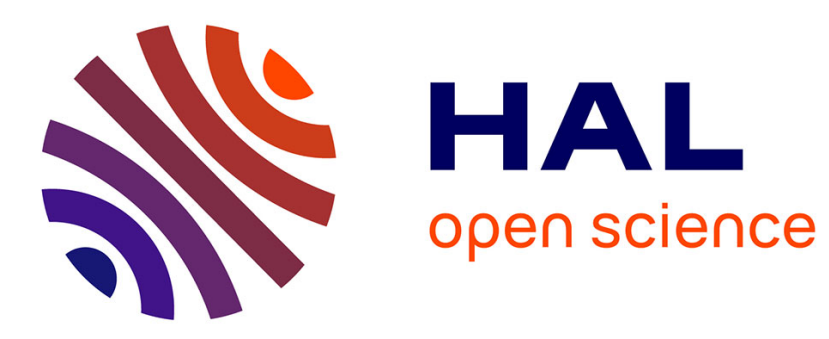

\title{
Mean field theory of directed polymers with random complex weights
}

\author{
Bernard Derrida, M. R. Evans, E. R. Speer
}

\section{To cite this version:}

Bernard Derrida, M. R. Evans, E. R. Speer. Mean field theory of directed polymers with random complex weights. Communications in Mathematical Physics, 1993, 156 (2), pp.221-244. 10.1007/BF02098482 . hal-03282964

\section{HAL Id: hal-03282964 \\ https://hal.science/hal-03282964}

Submitted on 19 Jul 2021

HAL is a multi-disciplinary open access archive for the deposit and dissemination of scientific research documents, whether they are published or not. The documents may come from teaching and research institutions in France or abroad, or from public or private research centers.
L'archive ouverte pluridisciplinaire HAL, est destinée au dépôt et à la diffusion de documents scientifiques de niveau recherche, publiés ou non, émanant des établissements d'enseignement et de recherche français ou étrangers, des laboratoires publics ou privés. 


\title{
Mean Field Theory of Directed Polymers with Random Complex Weights
}

\author{
B. Derrida ${ }^{1}$, M.R. Evans ${ }^{1}$, and E.R. Speer ${ }^{2}$ \\ ${ }^{1}$ Service de Physique Théorique, CEN Saclay, F91191 Gif-sur-Yvette, France \\ 2 School of Mathematics, Institute for Advanced Study, Princeton, NJ 08540, USA. Permanent \\ address: Department of Mathematics, Rutgers University, New Brunswick, NJ 08903, USA
}

Received September 29, 1992

\begin{abstract}
We show that for the problem of directed polymers on a tree with i.i.d. random complex weights on each bond, three possible phases can exist; the phase of a particular system is determined by the distribution $\rho$ of the random weights. For each of these three phases, we give the expression of the free energy per unit length in the limit of infinitely long polymers. Our proofs require several hypotheses on the distribution $\rho$, most importantly, that the amplitude and the phase of each complex weight be statistically independent. The main steps of our proofs use bounds on noninteger moments of the partition function and self averaging properties of the free energy. We illustrate our results by some examples and discuss possible generalizations to a larger class of distributions, to Random Energy Models, and to the finite dimensional case. We note that our results are not in agreement with the predictions of a recent replica approach to a similar problem.
\end{abstract}

\section{Introduction}

The behavior of directed polymers in a random medium has become over the last few years a central problem in the theory of disordered systems $[10,13,17,18,19$, $22,25,27]$. In its lattice version the problem can be formulated as follows: a random energy is assigned to each bond of the lattice, and every directed walk on the lattice has an energy given by the sum of all the bonds visited by the walk. As usual in statistical mechanics, the problem is to understand the thermal equilibrium of this system; in particular, we wish to calculate its partition function.

The problem is related to several physical phenomena: interfaces in two dimensional disordered magnets [14], the pinning of vortex lines by impurities, and the growth of the surfaces of Eden clusters and of ballistic deposits $[18,20]$. The problem also has many features in common with spin glasses, particularly at the mean field level [10]. (There are traditionally several ways of defining the mean field theory of a system in statistical mechanics. Here, as in [10], we will use this term to refer to the model of directed polymers in which the lattice is taken to be the Cayley tree.) This mean field case is so far one of the very few disordered systems for which it has been possible to prove that the predictions of the replica theory, in 
the case of broken symmetry between the replicas, give the correct free energy $[8,22,25]$.

Except in $1+1$ dimensions and in the mean field limit, the problem remains poorly understood from an analytical point of view. In dimension $1+1$ the equivalence of the KPZ equation [18] to the Burgers equation yields an exact knowledge of the critical exponents $[15,17]$. The mean field case, that is, the model on the Cayley tree, has been attacked by various techniques: travelling waves [3, 10], replicas [8], an analogy with random energy models [5], and martingales [2]. All these approaches lead to the same phase diagram and to the same expressions for the free energy in the different phases. At present, however, the last approach, based on the calculation of bounds of noninteger moments of the partition function $[2,11]$, is distinguished by its combination of simplicity and rigor.

In the original version of the directed polymer problem the weights assigned to the lattice bonds are positive. The problem may be generalized [5, 21, 24, 26, 28, 29] by removing this restriction: for example, the weights may have random signs or may be random complex variables. This generalization seems reasonable as a model for the hopping conductivity of strongly localized electrons, since the transmission of such electrons is dominated by directed paths, and interference effects are produced when the contributions of the individual paths are added.

The theory of directed polymers on the tree, with randomly chosen signs for the weights, was solved in [5] by generalizing the random energy model approach; it was pointed out there that the conclusions would be qualitatively the same for the case of complex weights. This approach predicted a phase diagram consisting of three different phases, called phases I, II, and III. Phases I and II are the two phases already present for positive weights; in that case they are respectively the high and low temperature phases (the replica approach predicts a broken replica symmetry in phase II). Phase III is a new high temperature phase, characterized by strong interference effects, which occurs when the flucutations in the phase (or sign) of the weights is large.

More recently, Goldschmidt and Blum [12] have used a replica approach to look at the problem of continuous directed polymers with complex weights in finite dimension. In the limit of high dimension, where it is expected that the results should coincide with those for the model on the tree, they found a phase diagram rather different from the one predicted by [5]. In particular, they obtained two additional phases (IV and V), corresponding to different schemes for the broken symmetry of the replicas. On the basis of these findings they questioned the validity of the results of [5].

Since neither of the two methods $[5,12]$ used so far for the study of the complex weights problem is rigorous, we believe that it is appropriate to attack the problem from a more mathematical point of view, in the hope of settling some of the open questions decisively. This is the motivation for the present paper.

Here, we extend the techniques based on the calculation of bounds of noninteger moments $([2,11])$ to obtain rigorously a complete description of the phase diagram in the case of complex weights (signed weights are a special case of this result). Our proof is valid when the amplitude and phase of the weight associated to each bond of the lattice are independent random variables. Under this hypothesis (which was present in the main example of [5] and in the whole of [12]), our results confirm the picture [5] of a phase diagram made up of three phases.

The balance of this paper is organised as follows. In Sect. 2 we define precisely the model we consider and summarise our main results, and in Sect. 3 we outline 
our strategy for the proof of these results. In Sect. 4 we obtain bounds on noninteger moments of the partition function, valid in phases I and III, as well as another moment bound which holds in all phases and is used in the study of phase II. In Sect. 5 we show that the free energy is partially self averaging in phases I and III; this concept, which will be defined precisely in Sect. 3, means essentially that the free energy is known with at least a nonzero probability. In Sect. 6 we prove that the partially self averaging property implies that free energy is self averaging; this completes the verification of our results for phases I and III. In Sect. 7 we use a monotonicity argument to obtain the free energy in phase II by reducing the problem to the known results from phase I and from the model with positive weights. Finally, in Sect. 8 we discuss possible extensions of our results to other models.

\section{Definition of the Model and Description of the Possible Phases}

In this section we define the model we are considering and describe the hypotheses which we make on the distribution of the weights associated to the bonds. We then present our main results on the possible phases of the system. Lastly we illustrate our results by simple examples.

The model we consider throughout this paper is that of directed polymers on the Cayley tree with branching ratio $d>1$ (or more precisely on one branch of this tree); the case $d=2$ is illustrated in Fig. 1 . On each bond $b$ of the tree there is a (possibly complex) weight $x_{b}$ randomly chosen according to a given probability distribution $\rho$. These weights are statistically independent.

There are $d^{L}$ walks of $L$ steps on this tree which start at the top and are directed downward. By definition, the weight $X_{W}$ of a walk $W$ is the product of the weights $x_{b}$ of all the bonds $b$ visited by the walk,

$$
X_{W}=\prod_{b \in W} x_{b}
$$

and the partition function $Z_{L}$ of walks of $L$ steps starting from the top of the tree is the sum of the weights of these walks,

$$
Z_{L}=\sum_{W} X_{W}
$$

Our goal is to predict the large $L$ behavior of $\log \left|Z_{L}\right| / L$.

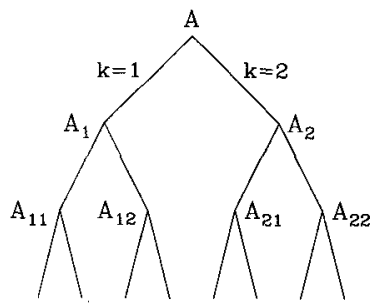

Fig. 1. A tree with branching ratio $d=2$ 
A useful way of attacking this problem is to write a recursion relation [10] for $Z_{L}$ :

$$
Z_{L}=\sum_{k=1}^{d} x_{k} Z_{L-1}^{(k)}, \quad \text { for } L \geqq 1 .
$$

Here the $x_{k}$ and the $Z_{L-1}^{(k)}$ are independent random variables, the $x_{k}$ have the distribution of the bond weights $x_{b}$, and the $Z_{L-1}^{(k)}$ have the distribution of $Z_{L-1}$; from the definition of $Z_{L}$, it is clear that $Z_{0}=1$. The recursion can easily be understood by grouping the walks appearing in (2.2) according to the direction of their first step: the contribution to $Z_{L}$ from all the walks making their first step in the $k^{\text {th }}$ direction is $x_{k} Z_{L-1}^{(k)}$, where $x_{k}$ is the weight of their common bond and $Z_{L-1}^{(k)}$ is the partition function of walks of length $L-1$ starting at point $A_{k}$ (see Fig. 1).

In the present paper we determine the limiting behavior of $\log \left|Z_{L}\right| / L$ completely, with some restrictions on the distribution $\rho$ of the bond weights. Throughout this paper we will make the following hypotheses about this distribution. We write $x$ for any random variable with distribution $\rho$ and $\langle\cdot\rangle$ for the average with respect to this distribution.

\section{Hypotheses on the Distribution $\rho$ :}

H1: The distribution of the amplitude $|x|$ is continuous, that is, for any real number $a, \operatorname{Prob}\{x=a\}=0$.

H2: The phase $s=x /|x|$ (well defined by H1) and the amplitude $|x|$ of $x$ are independent random variables.

H3: All moments $\left\langle|x|^{\alpha}\right\rangle$, with $\alpha>0$, are finite.

The most serious restriction here is the requirement of statistical independence between the phase and the amplitude. We believe that in fact the results below should remain true without this restriction; some further discussion of this point is given in Sect. 8.

We now turn to a discussion of the possible phases of this system. Let us first define the function $G(\alpha)$ for $\alpha>0$ by

$$
G(\alpha)=\frac{1}{\alpha} \log \left(d\left\langle|x|^{\alpha}\right\rangle\right)
$$

By $\mathrm{H} 3, G(\alpha)$ is finite for all $\alpha$; it is easy to check that $G$ is an infinitely differentiable, strictly convex function of $1 / \alpha$ (the latter is verified by calculating the second derivative of $G$ with respect to $1 / \alpha$ ) and hence has at most one minimum $\alpha_{\min }$. Since $d>1, G(\alpha)$ is decreasing for $\alpha$ sufficiently small; thus we may appropriately take $\alpha_{\min }$ to be infinite if $G(\alpha)$ has no minimum, and conclude that $G(\alpha)$ is strictly decreasing on the nonempty interval $0<\alpha<\alpha_{\min }$ and is strictly increasing for $\alpha>\alpha_{\min }$.

The system may exist in any of three phases, or lie on the common boundary of two or more of these. Distinct phases are characterized by distinct analytic expressions for the value of the free energy per step in the limit of infinitely long polymers. We begin by defining the various phases and will then state our results for the free energy. 
Definition. The phase of the system is determined as follows.

Phase I: The system is in phase I if

$$
\exists \alpha \in] 1,2] \text { such that } G(\alpha)<\log (d|\langle x\rangle|) .
$$

Phase II: The system is in phase II if

$$
\alpha_{\min }<1,
$$

or

$$
1 \leqq \alpha_{\min }<2 \text { and } G\left(\alpha_{\min }\right)>\log (d|\langle x\rangle|) .
$$

Phase III: The system is in phase III if

$$
\alpha_{\min }>2 \text { and } G(2)>\log (d|\langle x\rangle|) .
$$

We will say also that the system is on the $I-I I$ phase boundary if $1 \leqq \alpha_{\min }<2$ and $G\left(\alpha_{\min }\right)=\log (d|\langle x\rangle|)$, on the $I-I I I$ phase boundary if $\alpha_{\min }>2$ and $G(2)=\log (d|\langle x\rangle|)$, on the $I I-I I I$ phase boundary if $\alpha_{\min }=2$ and $\left.G(2)\right\rangle$ $\log (d|\langle x\rangle|)$, and at the triple point if $\alpha_{\min }=2$ and $G(2)=\log (d|\langle x\rangle|)$.

It is straightforward to verify that any system must be in precisely one phase, on precisely one phase boundary, or at the triple point, and that these possibilities are exclusive; the verification uses the properties of $G(\alpha)$ discussed above and the observation that

$$
\log (d|\langle x\rangle|)=\log (d\langle|x|\rangle)+\log |\langle s\rangle| \leqq G(1) .
$$

Note that equality holds in (2.8) only when $|\langle s\rangle|=1$, i.e., only when the phase variable $s$ does not fluctuate.

To describe the large $L$ behavior of the system we adopt the following terminolgy:

Definition. The free energy per step, $\log \left|Z_{L}\right| / L$, is self averaging to the value $f$ if it converges in probability to the constant $f$ as $L$ goes to infinity, that is, if for any $\varepsilon$, $\eta>0$ there exists a constant $L_{0}$ such that for $L>L_{0}$,

$$
\text { Prob }\left\{\left|\frac{\log \left|Z_{L}\right|}{L}-f\right|>\varepsilon\right\}<\eta \text {. }
$$

Now we may summarize the results of this paper as follows:

Phase $I$ : In phase $I, \log \left|Z_{L}\right| / L$ is self averaging to the value

$$
f_{\mathrm{I}} \equiv \log (d|\langle x\rangle|) \text {. }
$$

Phase $I I$ : In phase II, $\log \left|Z_{L}\right| / L$ is self averaging to the value

$$
f_{\mathrm{II}} \equiv G\left(\alpha_{\min }\right) \text {. }
$$

Phase III: In phase III, $\log \left|Z_{L}\right| / L$ is self averaging to the value

$$
f_{\mathrm{III}} \equiv G(2) \text {. }
$$

Moreover, these results hold also on the phase boundaries and at the triple point, where the corresponding limiting values of the free energy per unit length are easily seen to agree $\left(f_{\mathrm{I}}=f_{\mathrm{II}}\right.$ on the I-II phase boundary, etc.). 
It is easy to check, using the properties of $G(\alpha)$ mentioned above and (2.8), that (2.10)-(2.12) are equivalent to the statement that $\log \left|Z_{L}\right| / L$ is self averaging to the value $f$ given by

$$
f= \begin{cases}f_{\mathrm{II}}, & \text { when } \alpha_{\min } \leqq 1 \\ \max \left(f_{\mathrm{I}}, f_{\mathrm{II}}\right) & \text { when } 1 \leqq \alpha_{\min } \leqq 2 \\ \max \left(f_{\mathrm{I}}, f_{\mathrm{III}}\right), & \text { when } 2 \leqq \alpha_{\min }\end{cases}
$$

This is precisely the prediction of [5].

One should note that these results are not sensitive to details of the structure of the distribution of the phase variable $s$. In particular, $G(\alpha)$ is independent of this distribution, and the only way that the phase variable $s$ enters the criteria (2.5)-(2.7) and the expressions (2.10)-(2.13) is through the magnitude $|\langle s\rangle|$ of its average.

In the next section we shall outline the main steps to be followed in proving these results. Before this, however, let us close this section with a discussion of two examples.

Example 2.1. The Random Phase Problem. In the random phase problem, the weights $x_{b}$ have the form

$$
x_{b}=\exp \left(-\beta \varepsilon_{b}-i \gamma \varphi_{b}\right),
$$

where the energy $\varepsilon_{b}$ and phase $\varphi_{b}$ are real random variables with fixed distribution, and $\beta$ and $\gamma$ are parameters. For the purposes of exposition we will assume that $\varepsilon$ and $\varphi$ are independent Gaussian variables with respective densities

and

$$
\rho_{\text {energy }}(\varepsilon)=\frac{1}{\sqrt{2 \pi}} e^{-\varepsilon^{2} / 2}
$$

$$
\rho_{\text {phase }}(\varphi)=\frac{1}{\sqrt{2 \pi}} e^{-\varphi^{2} / 2} .
$$

These assumptions yield a lattice version, on the tree, of the continuum model considered in finite dimension in [12]. The parameter $\beta$ (the inverse temperature) allows one to tune the width of the distribution of the amplitude $|x|$ of $x$, and $\gamma$ plays a similar role for the phase variable $s$. The hypotheses $\mathrm{H} 1, \mathrm{H} 2$ and $\mathrm{H} 3$ are clearly satisfied for all $\beta>0$ and all real $\gamma$ (the model is invariant under the transformation $\gamma \rightarrow-\gamma$, so we consider only $\gamma \geqq 0$ ). For this choice of $\rho_{\text {energy }}$ the function (2.4) determining the phases is simply

$$
G(\alpha)=\frac{\log d}{\alpha}+\frac{\alpha \beta^{2}}{2},
$$

and thus $\alpha_{\min }=\beta_{0} / \beta$, where $\beta_{0}=(2 \log d)^{1 / 2}$. The phases throughout the $\beta, \gamma$ plane are determined by straightforward calculation from (2.5)-(2.7):

Phase I: The system is in phase I if

or

$$
\beta<\frac{\beta_{0}}{2} \text { and } \frac{\beta^{2}}{2}+\frac{\gamma^{2}}{2}<\frac{\beta_{0}^{2}}{4},
$$

$$
\frac{\beta_{0}}{2} \leqq \beta<\beta_{0} \text { and } \beta+\gamma<\beta_{0} .
$$


Phase II. The system is in phase II if

$$
\beta>\beta_{0}
$$

or

$$
\frac{\beta_{0}}{2}<\beta \leqq \beta_{0} \quad \text { and } \quad \beta+\gamma>\beta_{0}
$$

Phase III. The system is in phase III if

$$
\beta<\frac{\beta_{0}}{2} \text { and } \frac{\beta^{2}}{2}+\frac{\gamma^{2}}{2}>\frac{\beta_{0}^{2}}{4} .
$$

From (2.10)-(2.12) we find that in these three phases $\log \left(\left|Z_{L}\right|\right) / L$ is self averaging to the respective values $f_{\mathrm{I}}, f_{\mathrm{II}}$ and $f_{\mathrm{III}}$ given by:

$$
\begin{aligned}
& f_{\mathrm{I}}=\frac{\beta^{2}-\gamma^{2}}{2}+\frac{\beta_{0}^{2}}{2}, \\
& f_{\mathrm{II}}=\beta \beta_{0}, \\
& f_{\mathrm{III}}=\beta^{2}+\frac{\beta_{0}^{2}}{4} .
\end{aligned}
$$

The phase diagram is shown in Fig. 2. We note again that three phases exist for this model, in contrast to the five found in a replica calculation for a similar model in [12].

Example 2.2. The Random Sign Problem. In the random sign problem [5], the weight $x_{b}$ on the bond $b$ is given by

$$
x_{b}=s_{b} e^{-\beta \varepsilon_{b}},
$$

where $\varepsilon_{b}$ is an energy variable as above, which again we take for simplicity to have the Gaussian density $\rho_{\text {energy }}$ of (2.15), and $s_{b}$ is a sign variable with distribution

$$
s_{b}= \begin{cases}+1, & \text { with probability } 1-p, \\ -1, & \text { with probability } p .\end{cases}
$$

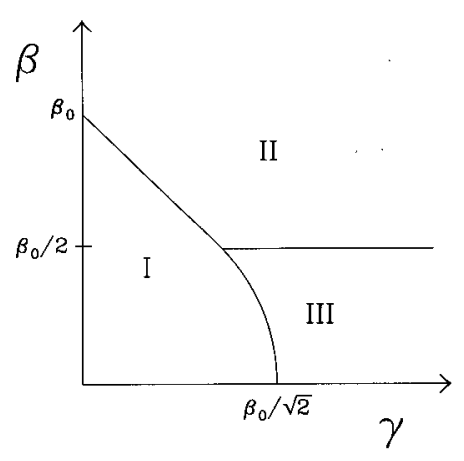

Fig. 2. The phase diagram for the random phase problem of Example 2.1, with Gaussian energies and Gaussian phases 
The parameters are now the inverse temperature $\beta \geqq 0$ and $p$; the obvious symmetry $p \rightarrow 1-p$ allows one to limit the discussion to $0 \leqq p \leqq 1 / 2$. $G(\alpha)$ is again given by (2.17). The conditions determining the phase diagram and the expressions for $\log \left|Z_{L}\right| / L$ in the various phases are the same as those $((2.18)-(2.21))$ in the random phase case above, but with $-\gamma^{2} / 2$ replaced by $\log |1-2 p|$ throughout (this follows from our earlier remark that the phase diagram and limiting free energy depend on $s_{b}$ only through its mean $\left.|\langle s\rangle|\right)$ and are identical to the predictions of [5]; we omit here the specific formulae, which are given in pages 969-970 of that reference.

\section{The Strategy of the Proof}

We now outline our strategy for proving that the free energy per unit length is self averaging to the values $f_{\mathrm{I}}, f_{\mathrm{II}}$, and $f_{\mathrm{III}}$ in phases, I, II, and III, respectively. The result for the I-III phase boundary will be obtained during our proof for phase III, and that for the I-II and II-III phase boundaries, and the triple point, during our proof for phase II; we omit any further details in this section.

For phases I and III, the arguments differ only in detail, and we summarize them together. In Sect. 4 we establish bounds on certain ratios of noninteger moments of the partition function (Theorem 4.1). In Sect. 5 we use these bounds to show (Theorem 5.2) that $\log \left|Z_{L}\right| / L$ satisfies a weaker version of the desired conclusion: that it is partially self averaging to the value $f_{\mathrm{I}}$ (respectively $f_{\mathrm{III}}$ ) in phase I (respectively phase III).

Definition. The free energy per step, $\log \left|Z_{L}\right| / L$, is partially self averaging to the value $f$ if (i) there exists a constant $a<1$ such that for any $\varepsilon>0$ there exists a constant $L_{0}$ such that for $L>L_{0}$,

$$
\operatorname{Prob}\left\{\frac{\log \left|Z_{L}\right|}{L}<f-\varepsilon\right\}<a,
$$

and (ii) for any $\varepsilon, \eta>0$ there exists a constant $L_{0}$ such that for $L>L_{0}$,

$$
\text { Prob }\left\{\frac{\log \left|Z_{L}\right|}{L}>f+\varepsilon\right\}<\eta \text {. }
$$

Speaking loosely, we might say that this definition asserts that, as $L \rightarrow \infty$, $\log \left|Z_{L}\right| / L$ is at most $f$ with probability one and is at least $f$ with some strictly positive probability $1-a$.

In Sect. 6 we prove (Theorem 6.4), for any distribution of the bond weights, that if $\log \left|Z_{L}\right| / L$ is partially self averaging then it is self averaging to the same value. It is here that the hypothesis that $|x|$ has continuous distribution is used. This, with the discussion of Sect. 5 noted above, completes the proof of our results in phases I and III.

Remark 3.1. It is instructive to compare our treatment of phases I and III with the treatment given in [2] of phase I in the positive weight case. The authors of [2] observe that when $Z_{L}$ is defined by the formula (2.2), $Z_{L} /\left\langle Z_{L}\right\rangle$ is a positive martingale, and that the martingale convergence theorem then implies that $Z_{L} /\left\langle Z_{L}\right\rangle$ converges almost surely, as $L \rightarrow \infty$, to some finite random variable $M$. Self averaging of $\log Z_{L} / L$ then follows if it can be shown that $M$ has no atom of 
mass at the origin. This is proved in two steps: first, it is shown that the event $\{M=0\}$ is a tail event and hence that $\operatorname{Prob}\{M=0\}$ is either 0 or 1 ; second, bounds on noninteger moments are used to show that this probability is less than one in phase I. We could, in fact, also use the martingale convergence theorem in phase I, although we have chosen to give a treatment that is uniform in phases I and III. An important difference is that, due to the possibility of cancellations in $Z_{L}$ caused by the presence of complex or negative weights, the event $\{M=0\}$ is no longer a tail event, and we need some other argument to show that these cancellations do not produce a mass in the distribution of $Z_{L}$, in the limit $L \rightarrow \infty$, which is concentrated on the value zero or even at values exponentially small compared to $\exp L f$, where $f=f_{\mathrm{I}}$ or $f_{\mathrm{III}}$. This is the argument that we give in Sect. 6 .

The proof for phase II is very different. The main idea we use is to vary the distribution of the phase variable $s$ while keeping the distribution of the amplitude $|x|$ fixed; this allows us to relate the distribution of $\log \left|Z_{L}\right| / L$ in phase II to that in phase I (near the I-II phase boundary) or to that in phase II of a system in which all the bond weights are positive. In this argument it is convenient to consider, along with the free energy $\log \left|Z_{L}\right|$, the auxiliary function $\log \left\langle\left\langle\left|Z_{L}\right|^{2}\right\rangle\right\rangle / 2$, where $\langle\langle\cdot\rangle\rangle$ denotes the average over all phase variables $s_{b}$, with the amplitude variables $\left|x_{b}\right|$ held fixed. This function is of use for two reasons. On the one hand, we will show that the asymptotic behavior of $\log \left\langle\left\langle\left|Z_{L}\right|^{2}\right\rangle\right\rangle / 2 L$ and of $\log \left|Z_{L}\right| / L$ is the same. In particular, in Sect. 4 we derive a moment bound (Theorem 4.2) which involves averages, over the phase variables $s_{b}$ only, of $\left|Z_{L}\right|^{2}$ and $\left|Z_{L}\right|^{4}$, and in Sect. 6 we use this bound to show that $\log \left\langle\left\langle\left|Z_{L}\right|^{2}\right\rangle\right\rangle / 2 L$ is self averaging to the value $f$ if and only if $\log \left|Z_{L}\right| / L$ is. On the other hand, $\left\langle\left\langle\left|Z_{L}\right|^{2}\right\rangle\right\rangle$ is naturally expressed as a sum of positive terms and is shown in Sect. 7 to be closely related to the free energy for walks on a tree with positive weights on the bonds. From known results for the latter model, and from our earlier results on the behavior of the system in phase I, we prove in Sect. 7 that $\log \left\langle\left\langle\left|Z_{L}\right|^{2}\right\rangle\right\rangle / 2 L$ is self averaging to $f_{\mathrm{II}}$ in phase II. This completes the proof of our result.

To finish this section, let us recall three well known inequalities which we shall use repeatedly in what follows. The first of these is valid whenever $0<\gamma \leqq 1$ and $t_{1}, t_{2}, \ldots$ are nonnegative:

$$
\left(\sum_{i} t_{i}\right)^{\gamma} \leqq \sum_{i}\left(t_{i}\right)^{\gamma} .
$$

The others are special cases of Jensen's inequality: if $y$ is a nonnegative random variable, then

$$
\begin{array}{ll}
\left\langle y^{\gamma}\right\rangle \leqq\langle y\rangle^{\gamma}, & \text { if } 0<\gamma \leqq 1, \\
\left\langle y^{\gamma}\right\rangle \geqq\langle y\rangle^{\gamma}, & \text { if } 1 \leqq \gamma .
\end{array}
$$

\section{Moment Formulas and Bounds}

In this section we obtain explicit and asymptotic formulas for the first and second moments of the partition function $Z_{L}$, as well as bounds (Theorem 4.1) for certain ratios of its noninteger moments. These results will be used in Sect. 5 to show that in phases I and III, and on the I-III phase boundary, $\log \left|Z_{L}\right| / L$ is partially selfaveraging to the value $f_{\mathrm{I}}$ or $f_{\mathrm{III}}$. We also derive a bound (Theorem 4.2) on the ratio 
$\left\langle\left\langle\left|Z_{L}\right|^{4}\right\rangle\right\rangle /\left\langle\left\langle\left|Z_{L}\right|^{2}\right\rangle\right\rangle^{2}$, which will be used in Sect. 6 to relate the behavior of $\log \left|Z_{L}\right| / L$ and $\log \left\langle\left\langle\left|Z_{L}\right|^{2}\right\rangle\right\rangle / 2 L$.

The calculation of the first two integer moments of the partition function can be done directly from the recursion (2.3), which yields

$$
\left\langle Z_{L+1}\right\rangle=d\langle x\rangle\left\langle Z_{L}\right\rangle \text {, }
$$

and then

$$
\left|Z_{L+1}^{2}\right|=\sum_{1 \leqq k \leqq d}\left|x_{k} Z_{L}^{(k)}\right|^{2}+\sum_{1 \leqq j \neq k \leqq d} x_{j} Z_{L}^{(j)} x_{k}^{*} Z_{L}^{(k) *},
$$

(where $*$ denotes the complex conjugate) so that

$$
\left\langle\left|Z_{L+1}^{2}\right|\right\rangle=d\left\langle|x|^{2}\right\rangle\left\langle\left|Z_{L}\right|^{2}\right\rangle+d(d-1)|\langle x\rangle|^{2}\left|\left\langle Z_{L}\right\rangle\right|^{2} .
$$

Then an induction on $L$ yields

$$
\left\langle Z_{L}\right\rangle=[d\langle x\rangle]^{L}
$$

and, with $a=d\left\langle\left|x^{2}\right|\right\rangle$ and $b=|d\langle x\rangle|^{2}$,

$$
\left\langle\left|Z_{L}^{2}\right|\right\rangle=\frac{a^{L}}{d}+\left(\frac{d-1}{d}\right)\left(\frac{a^{L+1}-b^{L+1}}{a-b}\right) .
$$

Thus if $a>b$, which is always true in phase III (see (2.7)), $\left\langle\left|Z_{L}^{2}\right|\right\rangle$ grows as $a^{L}$ :

$$
\lim _{L \rightarrow \infty} \frac{\left\langle\left|Z_{L}^{2}\right|\right\rangle}{\left(d\left\langle\left|x^{2}\right|\right\rangle\right)^{L}}=\frac{1}{d}+\frac{(d-1) a}{d(a-b)} ;
$$

while if $a=b$, as on the I-III phase boundary, $\left\langle\left|Z_{L}^{2}\right|\right\rangle$ grows as $L a^{L}$ :

$$
\lim _{L \rightarrow \infty} \frac{\left\langle\left|Z_{L}^{2}\right|\right\rangle}{L\left(d\left\langle\left|x^{2}\right|\right\rangle\right)^{L}}=\frac{d-1}{d} .
$$

Note that the ratio $\left\langle\left|Z_{L}^{2}\right|\right\rangle /\left|\left\langle Z_{L}\right\rangle\right|^{2}$ remains bounded when the system satisfies the condition (2.5) for phase I, and also the condition $\alpha_{\min }>2$, since then $a<b$. This could be exploited to show that $\log \left|Z_{L}\right| / L$ is partially self averaging to $f_{\mathrm{I}}$ under these conditions, but sharper results valid whenever the system is in phase I may be obtained from the next theorem, which gives bounds on ratios of non-integer moments of $Z_{L}$.

Theorem 4.1. (a) In phase I, there exists an $\alpha$ satisfying $1<\alpha \leqq 2$ and a constant $B_{\alpha}$ such that for all $L \geqq 0$,

$$
\frac{\left\langle\left|Z_{L}\right|^{\alpha}\right\rangle}{\left\langle\left|Z_{L}\right|\right\rangle^{\alpha}} \leqq B_{\alpha}
$$

(b) Whenever $\alpha_{\min }>2$, and in particular in phase III and on the I-III phase boundary, there exists an $\alpha>2$ and a constant $\widetilde{B}_{\alpha}$ such that for all $L \geqq 0$,

$$
\frac{\left\langle\left|Z_{L}\right|^{\alpha}\right\rangle}{\left\langle\left|Z_{L}\right|^{2}\right\rangle^{\alpha / 2}} \leqq \tilde{B}_{\alpha}
$$

Proof. (a) Recall that $\langle\langle Y\rangle\rangle$ denotes the average of the quantity $Y$ over the phase variables $s_{b}=x_{b} /\left|x_{b}\right|$. For $\alpha \leqq 2$, Jensen's inequality (3.4) implies that

$$
\left\langle\left|Z_{L}\right|^{\alpha}\right\rangle=\left\langle\left(\left|Z_{L}\right|^{2}\right)^{\alpha / 2}\right\rangle \leqq\left\langle\left\langle\left\langle\left. Z_{L}\right|^{2}\right\rangle\right\rangle^{\alpha / 2}\right\rangle,
$$


and since $\left\langle\left|Z_{L}\right|\right\rangle \geqq\left|\left\langle Z_{L}\right\rangle\right|$, to establish (4.8) it suffices to give a bound for the ratio $R_{L} \equiv\left\langle\left\langle\left\langle\left|Z_{L}\right|^{2}\right\rangle\right\rangle^{\alpha / 2}\right\rangle /\left|\left\langle Z_{L}\right\rangle\right|^{\alpha}$. From (4.2),

$$
\begin{aligned}
\left\langle\left\langle\left\langle Z_{L+1}^{2} \mid\right\rangle\right\rangle^{\alpha / 2}\right\rangle= & \left\langle\left[\sum_{1 \leqq k \leqq d}\left|x_{k}\right|^{2}\left\langle\left\langle\left|Z_{L}^{(k)}\right|^{2}\right\rangle\right\rangle\right.\right. \\
& \left.\left.+\sum_{1 \leqq j \neq k \leqq d}|\langle s\rangle|^{2}\left|x_{k}\right|\left|x_{j}\right|\left\langle\left\langle Z_{L}^{(k)}\right\rangle\right\rangle\left\langle\left\langle Z_{L}^{(j) *}\right\rangle\right\rangle\right]^{\alpha / 2}\right\rangle .
\end{aligned}
$$

Clearly $\left\langle\left\langle Z_{L}\right\rangle\right\rangle$ has phase $\langle s\rangle^{L}$ and hence $\left\langle\left\langle Z_{L}^{\prime}\right\rangle\right\rangle\left\langle\left\langle Z_{L}^{\prime \prime}\right\rangle\right\rangle \geqq 0$ whenever $Z_{L}^{\prime}$ and $Z_{L}^{\prime \prime}$ have the distribution of $Z_{L}$. Therefore the second term in (4.11) is positive and hence by the inequality (3.3) and another application of Jensen's inequality (3.4),

$$
\begin{aligned}
\left\langle\left\langle\left\langle\left|Z_{L+1}^{2}\right|\right\rangle\right\rangle^{\alpha / 2}\right\rangle \leqq & \left\langle\sum_{1 \leqq k \leqq d}\left|x_{k}\right|^{\alpha}\left\langle\left\langle\left|Z_{L}^{(k)}\right|^{2}\right\rangle\right\rangle^{\alpha / 2}\right. \\
& +\left[\sum_{1 \leqq j \neq k \leqq d}|\langle s\rangle|^{2}\left|x_{k}\right|\left|x_{j}\right|\left\langle\left\langle Z_{L}^{(k)}\right\rangle\right\rangle\left\langle\left\langle Z_{L}^{(j) *}\right\rangle\right]^{\alpha / 2}\right\rangle \\
\leqq & d\left\langle|x|^{\alpha}\right\rangle\left\langle\left\langle\left\langle\left|Z_{L}\right|^{2}\right\rangle\right\rangle^{\alpha / 2}\right\rangle+[d(d-1)]^{\alpha / 2}|\langle x\rangle|^{\alpha}\left|\left\langle Z_{L}\right\rangle\right|^{\alpha},
\end{aligned}
$$

so that, from (4.1),

$$
R_{L+1} \leqq \frac{d\left\langle|x|^{\alpha}\right\rangle}{(d|\langle x\rangle|)^{\alpha}} R_{L}+\left(\frac{d-1}{d}\right)^{\alpha / 2} .
$$

Now in phase I (see (2.5)) there exists $\alpha \in] 1,2]$ for which $d\left\langle|x|^{\alpha}\right\rangle /(d|\langle x\rangle|)^{\alpha}<1$; with this value of $\alpha, R_{L}$ will remain finite as $L \rightarrow \infty$ (recall that $\alpha \leqq 2$ was necessary for the use of (3.3) and Jensen's inequality (3.4)). Thus (4.8) holds with $B_{\alpha}$ given by

$$
B_{\alpha}=\left(\frac{d-1}{d}\right)^{\alpha / 2}\left(1-\frac{d\left\langle|x|^{\alpha}\right\rangle}{|d\langle x\rangle|^{\alpha}}\right)^{-1} .
$$

(b) We now must estimate $R_{L}^{\prime} \equiv\left\langle\left|Z_{L}\right|^{\alpha}\right\rangle /\left\langle\left|Z_{L}\right|^{2}\right\rangle^{\alpha / 2}$ for $\left.\alpha_{\min }\right\rangle 2$. From (2.3) we have

$$
\begin{aligned}
\left|Z_{L+1}\right|^{4} \leqq & \sum_{k}\left|x_{k} Z_{L}^{(k)}\right|^{4}+3 \sum_{j \neq k}\left|x_{j} Z_{L}^{(j)}\right|^{2}\left|x_{k} Z_{L}^{(k)}\right|^{2} \\
& +4 \sum_{j \neq k}\left|x_{j} Z_{L}^{(j)}\right|\left|x_{k} Z_{L}^{(k)}\right|^{3}+6 \sum_{j \neq k \neq l}\left|x_{j} Z_{L}^{(j)}\right|^{2}\left|x_{k} Z_{L}^{(k)}\right|\left|x_{l} Z_{L}^{(l)}\right| \\
& +\sum_{i \neq j \neq k \neq l}\left|x_{i} Z_{L}^{(i)}\right|\left|x_{j} Z_{L}^{(j)}\right|\left|x_{k} Z_{L}^{(k)}\right|\left|x_{l} Z_{L}^{(l)}\right|
\end{aligned}
$$

Now choose $\alpha$ satisfying $\alpha \leqq 8 / 3$, raise (4.15) to the power $\alpha / 4$, apply the inequality (3.3), treating the right-hand side as a sum of $d^{4}$ terms (in contrast to what was done in passing from (4.11) to (4.12)) and take the expectation, to yield

$$
\begin{aligned}
\left\langle\left|Z_{L+1}\right|^{\alpha}\right\rangle \leqq & d\left\langle|x|^{\alpha}\right\rangle\left\langle\left|Z_{L}\right|^{\alpha}\right\rangle+3 d(d-1)\left\langle|x|^{\alpha / 2}\right\rangle^{2}\left\langle\left|Z_{L}\right|^{\alpha / 2}\right\rangle^{2} \\
& +4 d(d-1)\left\langle|x|^{\alpha / 4}\right\rangle\left\langle|x|^{3 \alpha / 4}\right\rangle\left\langle\left|Z_{L}\right|^{\alpha / 4}\right\rangle\left\langle\left|Z_{L}\right|^{3 \alpha / 4}\right\rangle \\
& +6 d(d-1)(d-2)\left\langle|x|^{\alpha / 4}\right\rangle^{2}\left\langle|x|^{\alpha / 2}\right\rangle\left\langle\left|Z_{L}\right|^{\alpha / 4}\right\rangle^{2}\left\langle\left|Z_{L}\right|^{\alpha / 2}\right\rangle \\
& +d(d-1)(d-2)(d-3)\left\langle|x|^{\alpha / 4}\right\rangle^{4}\left\langle\left|Z_{L}\right|^{\alpha / 4}\right\rangle .
\end{aligned}
$$


Now Jensen's inequality (3.4), with the condition $\alpha \leqq 8 / 3$, implies that

$$
\left\langle|x|^{\gamma}\right\rangle \leqq\left\langle|x|^{2}\right\rangle^{\gamma / 2} \text { and }\left\langle\left|Z_{L}\right|^{\gamma}\right\rangle \leqq\left\langle\left|Z_{L}\right|^{2}\right\rangle^{\gamma / 2} \text {, }
$$

for $\gamma=\alpha / 4, \alpha / 2$, and $3 \alpha / 4$. Moreover, from (4.3), $\left\langle\left|Z_{L+1}\right|^{2}\right\rangle \geqq d\left\langle\left|x^{2}\right|\right\rangle\left\langle\left|Z_{L}\right|^{2}\right\rangle$. Thus (4.16) yields

$$
R_{L+1}^{\prime} \leqq \frac{d\left\langle|x|^{\alpha}\right\rangle}{\left(d\left\langle|x|^{2}\right\rangle\right)^{\alpha / 2}} R_{L}^{\prime}+\frac{d^{4}-d}{d^{\alpha / 2}} .
$$

Since by hypothesis $\alpha_{\min }>2$, we may choose $\alpha$ in the interval ]2, 8/3[ so that $G(2)>G(\alpha)$, i.e., so that the ratio $d\left\langle|x|^{\alpha}\right\rangle /\left(d\left\langle|x|^{2}\right\rangle\right)^{\alpha / 2}$ is less than 1 . With this choice of $\alpha, R_{L}^{\prime}$ is bounded and (4.9) holds with

$$
\tilde{B}_{\alpha}=\frac{d^{4}-d}{d^{\alpha / 2}}\left(1-\frac{d\left\langle|x|^{\alpha}\right\rangle}{\left(d\left\langle|x|^{2}\right\rangle\right)^{\alpha / 2}}\right)^{-1} .
$$

The next theorem holds in all phases, that is, for any bond weight distribution satisfying the hypotheses $\mathrm{H} 1-\mathrm{H} 3$.

Theorem 4.2. The ratio $\left\langle\left\langle\left|Z_{L}\right|^{4}\right\rangle\right\rangle /\left\langle\left\langle\left. Z_{L}\right|^{2}\right\rangle\right\rangle^{2}$ is bounded as a function of $L$ :

$$
\frac{\left\langle\left\langle\left|Z_{L}\right|^{4}\right\rangle\right\rangle}{\left\langle\left\langle\left|Z_{L}\right|^{2}\right\rangle\right\rangle^{2}} \leqq 24
$$

Proof. If we denote by $S_{W}=X_{W} /\left|X_{W}\right|$ the phase associated to the walk $W$, then we can write from the definition (2.2) of $Z_{L}$,

$$
\left\langle\left\langle\left|Z_{L}\right|^{4}\right\rangle\right\rangle=\sum_{W_{1}, W_{2}, W_{3}, W_{4}}\left|X_{W_{1}} X_{W_{2}} X_{W_{3}} X_{W_{4}}\right|\left\langle S_{W_{1}} S_{W_{2}}^{*} S_{W_{3}} S_{W_{4}}^{*}\right\rangle
$$

and

$$
\left\langle\left\langle\left|Z_{L}\right|^{2}\right\rangle\right\rangle^{2}=\sum_{W_{1}, W_{2}, W_{3}, W_{4}}\left|X_{W_{1}} X_{W_{2}} X_{W_{3}} X_{W_{4}}\right|\left\langle S_{W_{1}} S_{W_{2}}^{*}\right\rangle\left\langle S_{W_{3}} S_{W_{4}}^{*}\right\rangle .
$$

Under a permutation $\left(W_{1}, W_{2}, W_{3}, W_{4}\right) \rightarrow\left(W_{P(1)}, W_{P(2)}, W_{P(3)}, W_{P(4)}\right)$ of the four walks the amplitude $\left|X_{W_{1}} X_{W_{2}} X_{W_{3}} X_{W_{4}}\right|$ remains unchanged, and thus one can write (4.21) and (4.22) as

$$
\left\langle\left\langle\left|Z_{L}\right|^{4}\right\rangle\right\rangle=\sum_{W_{1}, W_{2}, W_{3}, W_{4}}\left|X_{W_{1}} X_{W_{2}} X_{W_{3}} X_{W_{4}}\right| \frac{1}{24} \sum_{P}\left\langle S_{W_{P(1)}} S_{W_{P(2)}}^{*} S_{W_{P(3)}} S_{W_{P(4)}}^{*}\right\rangle
$$

and

$$
\left\langle\left\langle\left|Z_{L}\right|^{2}\right\rangle\right\rangle^{2}=\sum_{W_{1}, W_{2}, W_{3}, W_{4}}\left|X_{W_{1}} X_{W_{2}} X_{W_{3}} X_{W_{4}}\right| \frac{1}{24} \sum_{P}\left\langle S_{W_{P(1)}} S_{W_{P(2)}}^{*}\right\rangle\left\langle S_{W_{P(3)}} S_{W_{P(4)}}^{*}\right\rangle .
$$

Now for every choice $W_{1}, W_{2}, W_{3}, W_{4}$ of walks there is a permutation $Q$ for which

$$
\begin{aligned}
\left\langle S_{W_{Q(1)}} S_{W_{Q(2)}}^{*} S_{W_{Q(3)}} S_{W_{Q(4)}}^{*}\right\rangle & =\left\langle S_{W_{Q(1)}} S_{W_{Q(2)}}^{*}\right\rangle\left\langle S_{W_{Q(3)}} S_{W_{Q(4)}}^{*}\right\rangle \\
& =|\langle s\rangle|^{m\left(W_{1}, W_{2}, W_{3}, W_{4}\right)},
\end{aligned}
$$


where $m\left(W_{1}, W_{2}, W_{3}, W_{4}\right)$ is the number of bonds on the lattice which are visited by an odd number of the four walks. Moreover, (4.25) maximizes $\left|\left\langle S_{W_{P(1)}} S_{W_{P(2)}}^{*} S_{W_{P(3)}} S_{W_{P(4)}}^{*}\right\rangle\right|$ among all permutations $P$, so that from (4.23),

$$
\left\langle\left\langle\left|Z_{L}\right|^{4}\right\rangle\right\rangle \leqq \sum_{W_{1}, W_{2}, W_{3}, W_{4}}\left|X_{W_{1}} X_{W_{2}} X_{W_{3}} X_{W_{4}}\right||\langle s\rangle|^{m\left(W_{1}, W_{2}, W_{3}, W_{4}\right)},
$$

and using the fact that $\left\langle S_{W_{P(1)}} S_{W_{P(2)}}^{*}\right\rangle\left\langle S_{W_{P(3)}} S_{W_{P(1)}}^{*}\right\rangle \geqq 0$ for all $P$, one gets from (4.24),

$$
\left\langle\left\langle\left|Z_{L}\right|^{2}\right\rangle\right\rangle^{2} \geqq \frac{1}{24} \sum_{W_{1}, W_{2}, W_{3}, W_{4}}\left|X_{W_{1}} X_{W_{2}} X_{W_{3}} X_{W_{4}}\right||\langle s\rangle|^{m\left(W_{1}, W_{2}, W_{3}, W_{4}\right)} .
$$

Theorem 4.2 follows by taking the ratio of (4.26) and (4.27).

Remark 4.3. By a longer argument one can improve the estimate of the theorem by showing that the ratio in (4.20) is at most 3 . Any finite bound suffices in what follows.

\section{Partial Self Averaging of the Free Energy in Phases I and III}

In this section we prove (Theorem 5.2) that $\log \left|Z_{L}\right| / L$ is partially self averaging to $f_{\mathrm{I}}$ and $f_{\mathrm{III}}$ in phases I and III, respectively, and to $f_{\mathrm{I}}=f_{\mathrm{III}}$ on the I-III phase boundary. The proof is based on Theorem 4.1 and the following lemma.

Lemma 5.1. Suppose that the nonnegative random variable $Y$ satisfies $\left\langle Y^{\gamma}\right\rangle\langle Y\rangle^{\gamma}$ $\leqq B$ for some constant $B \geqq 1$ and for some $\gamma>1$. Then:

(a) For any $\eta>0$ there is an $M \geqq 1$ such that

$$
\operatorname{Prob}\left\{\frac{Y}{\langle Y\rangle}<M\right\} \geqq 1-\eta ;
$$

(b) For any $\eta^{\prime}>0$ there is an $m$, with $0<m \leqq 1$, such that

$$
\operatorname{Prob}\left\{\frac{Y}{\langle Y\rangle}>m\right\} \geqq \frac{1-\eta^{\prime}}{B^{1 /(\gamma-1)}} .
$$

Here $M$ and $m$ may depend on $\gamma$ and $B$ and on $\eta$ and $\eta^{\prime}$, respectively, but not otherwise on the distribution of $Y$.

Proof. (a) The result follows immediately from the generalized Chebyshev inequality, which yields $\operatorname{Prob}\{Y /\langle Y\rangle \geqq M\} \leqq B / M^{\gamma}$. Of course, the conclusion of (a) holds whenever $\langle Y\rangle\langle\infty$.

(b) Let $\mu$ be the measure on $[0, \infty[$ describing the distribution of $Y$, so that $\mu([a, b[)=\operatorname{Prob}\{a \leqq Y<b\}$. Fix $m>0$, let $A=\mu([0, m<Y>])$ be the probability that $Y \leqq m\langle Y\rangle$, and let $\hat{\mu}$ and $\tilde{\mu}$ be the restrictions of $\mu$ to $[0, m\langle Y\rangle]$ and ]$m\langle Y\rangle, \infty[$, respectively, normalized as probability measures, so that $\mu=A \hat{\mu}+$ $(1-A) \tilde{\mu}$. Finally, let $\tilde{Y}$ be the random variable whose distribution is specified by $\tilde{\mu}$. Then

$$
\langle Y\rangle=A \int_{0}^{m\langle Y\rangle} y d \hat{\mu}+(1-A) \int_{m\langle Y\rangle}^{\infty} y d \tilde{\mu} \leqq A m\langle Y\rangle+(1-A)\langle\tilde{Y}\rangle,
$$


so that

$$
\langle Y\rangle \leqq \frac{1-A}{1-m A}\langle\tilde{Y}\rangle,
$$

and

$$
\left\langle Y^{\dot{\gamma}}\right\rangle=A \int_{0}^{m\langle\boldsymbol{Y}\rangle} y^{\gamma} d \hat{\mu}+(1-A) \int_{m\langle Y\rangle}^{\infty} y^{\gamma} d \tilde{\mu} \geqq(1-A)\left\langle\tilde{Y}^{\gamma}\right\rangle .
$$

Since $\left\langle\tilde{Y}^{\gamma}\right\rangle \geqq\langle\tilde{Y}\rangle^{\gamma}$ by Jensen's inequality (3.5), we conclude that

$$
B \geqq\left\langle Y^{\gamma}\right\rangle /\langle Y\rangle^{\gamma} \geqq(1-A)^{1-\gamma}(1-m A)^{\gamma},
$$

or, since $1-m A \geqq 1-m$,

$$
\operatorname{Prob}\left\{\frac{Y}{\langle Y\rangle}>m\right\}=1-A \geqq \frac{(1-m)^{\gamma /(\gamma-1)}}{B^{1 /(\gamma-1)}} .
$$

Thus (5.2) is satisfied for sufficiently small $m$.

Using this lemma we can prove the main result of this section:

Theorem 5.2. $\log \left|Z_{L}\right| / L$ is partially self averaging to $f_{\mathrm{I}}=\log (d|\langle x\rangle|)$ in phase $\mathrm{I}$, to $f_{\mathrm{III}}=\log \left(d\left\langle|x|^{2}\right\rangle\right) / 2$ in phase III, and to $f_{\mathrm{I}}=f_{\mathrm{III}}$ on the I-III phase boundary.

Proof. The result is a direct consequence of Theorem 4.1, the asymptotic (or exact) formulae (4.4), (4.6), and (4.7), and the preceding lemma. The proof is similar in phase I and in phase III; we illustrate it by discussing the cases in which the system is in phase III or on the I-III phase boundary, and first verify (3.2). Given $\eta, \varepsilon>0$ we apply Lemma 5.1 (a) with $Y=\left|Z_{L}^{2}\right|$ and $\gamma=\alpha / 2, B=\widetilde{B}_{\alpha}$, where $\alpha, \widetilde{B}_{\alpha}$ are the constants of (4.9), to conclude that

$$
\text { Prob }\left\{\frac{\log \left|Z_{L}\right|^{2}}{2 L}>\frac{\log \left\langle\left|Z_{L}^{2}\right|\right\rangle+\log M}{2 L}\right\}<\eta,
$$

for some $M \geqq 1$ and for all $L$. If we now choose $L$ so large that $\log M / 2 L<\varepsilon / 2$ and, using (4.6) in phase III and (4.7) on the I-III boundary, that $\log \left\langle\left|Z_{L}^{2}\right|\right\rangle / 2 L$ $<f_{\text {III }}+\varepsilon / 2$, then (3.2) will hold with $f=f_{\text {III }}$. To verify (3.1) we suppose given $\varepsilon>0$ and, with $Y$, $\gamma$, and $B$ as in the argument for (5.8) above and with $\eta^{\prime}=1 / 2$, conclude from Lemma 5.1(b) that

$$
\operatorname{Prob}\left\{\frac{\log \left|Z_{L}\right|^{2}}{2 L}<\frac{\log \left\langle\left|Z_{L}^{2}\right|\right\rangle+\log m}{2 L}\right\}<\frac{1}{2 \tilde{B}_{\alpha}^{2 /(\alpha-2)}},
$$

for some $m, 0<m \leqq 1$, and for all $L$. We now choose $L$ large enough that $|\log m| / 2 L<\varepsilon / 2$; an argument as before then leads to $(3.1)$, with $a=1 / 2 \widetilde{B}_{\alpha}^{2 /(\alpha-2)}$.

\section{Partial Self Averaging Implies Self Averaging}

Our main goal in this section is to prove that if $\log \left|Z_{L}\right| / L$ is partially self averaging then it is self averaging to the same value; this result holds independently of the phase of the system. Our proof is based on the recursion (2.3) for $Z_{L}$ and is inspired by an argument given by [6]. When combined with Theorem 5.2 , this will complete 
the proof of the results discussed in Sect. 2 for phases I and III. We will also prove a result needed for our discussion of phase II in Sect. 7: that $\log \left|Z_{L}\right| / L$ is self averaging if and only if $\log \left\langle\left\langle\left|Z_{L}^{2}\right|\right\rangle\right\rangle / 2 L$ is.

Let us begin by introducing some notation and establishing two preliminary results. Given complex numbers $z^{(1)}, \ldots, z^{(d)}$, we write $\underline{z}=\left(z^{(1)}, \ldots, z^{(d)}\right)$ and $\|\underline{z}\|=\max _{1 \leqq k \leqq d}\left|z^{(k)}\right|$, and for $\|z\| \neq 0$ define

$$
q\left(\underline{z}^{\prime}, \underline{z}\right)=\frac{\sum_{k=1}^{d} z^{\prime(k)} z^{(k)}}{\|\underline{z}\|} .
$$

Lemma 6.1. Let $x^{(1)}, \ldots, x^{(d)}$ be independent, identically distributed complex random variables such that $\left|x^{(k)}\right|$ has continuous distribution. Then for any $v>0$, there exists a $c>0$ such that, for any $\underline{z} \in \mathbb{C}^{d}$ with $\left(z^{(1)}, \ldots, z^{(d)}\right)$ not all zero,

$$
\operatorname{Prob}\{|q(\underline{x}, \underline{z})|<c\}<v .
$$

Proof. The function $q$ is symmetric in the variables $\left(z^{(1)}, \ldots, z^{(d)}\right)$ so that it is no loss of generality to assume that $z^{(1)}$ has the largest value among these; since not all of the $z^{(j)}$ vanish, $\left|z^{(1)}\right|=\|\underline{z}\|>0$. Then

$$
\begin{aligned}
\operatorname{Prob}\{|q(\underline{x}, \underline{z})|<c\} & =\operatorname{Prob}\left\{\left|x^{(1)}+\sum_{k=2}^{d} \frac{x^{(k)} z^{(k)}}{\left|z^{(1)}\right|}\right|<c\right\} \\
& \leqq \operatorname{Prob}\left\{|| x^{(1)}|-| \sum_{k=2}^{d} \frac{x^{(k)} z^{(k)}}{\left|z^{(1)}\right|}||<c\right\} .
\end{aligned}
$$

Now suppose that $v>0$. We claim that if $c$ is sufficiently small, then for any real number $a$,

$$
\operatorname{Prob}\left\{|| x^{(1)}|-a|<c\right\}<v .
$$

If this is true, then (6.2) follows from (6.3) by taking $a=\left|\sum_{k=2}^{d} z^{(k)} x^{(k)}\right| /\left|z^{(1)}\right|$ and integrating over the distribution of $x^{(2)}, \ldots, x^{(d)}$. To prove the claim let $F(t) \equiv \operatorname{Prob}\left\{\left|x^{(1)}\right| \leqq t\right\} ; F$ is continuous by hypothesis $\mathrm{H} 3$ and has the finite limit 1 as $t \rightarrow \infty$, so $F$ is uniformly continuous on $\mathbb{R}$. This in turn means that we may choose $c$ so that if $t^{\prime} \leqq t$ and $\left|t-t^{\prime}\right|<2 c$, then $F(t)-F\left(t^{\prime}\right)=\operatorname{Prob}\left\{t^{\prime}<\left|x^{(1)}\right| \leqq t\right\}$ $<v$, and this implies (6.4).

We next show how the previous estimate may be combined with the recursive definition (2.3) to prove that, if $\operatorname{Prob}\left\{\log \left|Z_{L}\right| / L<f\right\}$ is uniformly less than one for all sufficiently large $L$, then this probability will be arbitrarily small for all sufficiently large $L$. Let us begin with the following remark:

Remark 6.2. Suppose that the parameter $v$ satisfies $0<v<1$ and that the map $\phi_{v}:\left[0, \infty\left[\rightarrow\left[0, \infty\left[\right.\right.\right.\right.$ is defined by $\phi_{v}(t)=t^{d}+v$. Then for sufficiently small $v, \phi_{v}$ has two fixed points $t_{v}^{ \pm}$which satisfy $0<t_{v}^{-}<t_{v}^{+}<1$ and $\lim _{v \rightarrow 0} t_{v}^{-}=0$, $\lim _{v \rightarrow 0} t_{v}^{+}=1$. Moreover, for $0 \leqq t<t_{v}^{+}, \lim _{k \rightarrow \infty} \phi_{v}^{k}(t)=t_{v}^{-}$.

Lemma 6.3. Suppose that for some $L$ there exist real numbers $F$ and a, with $0<a<1$, such that

$$
\operatorname{Prob}\left\{\log \left|Z_{L}\right| \leqq F\right\}<a
$$


Then for any $\eta>0$ there exist a positive integer $k$ and a real number $A$, depending only on $a$ and $\eta$, such that

$$
\operatorname{Prob}\left\{\log \left|Z_{L+k}\right| \leqq F-A\right\}<\eta .
$$

Proof. Choose $v>0$ small enough so that $\phi_{v}$ has the properties summarized in Remark 6.3, and so that $t_{v}^{+}>a$ and $t_{v}^{-}<\eta / 2$. By Lemma 6.1, there exists a constant $C$ such that

$$
\operatorname{Prob}\{\log |q(\underline{x}, \underline{z})|<-C\}<v,
$$

uniformly in $\underline{z}$. ( $C=-\log c$ in the notation of Lemma 6.1 and will typically be positive.) We will show that for all $j \geqq 0$,

$$
\operatorname{Prob}\left\{\log \left|Z_{L+j}\right| \leqq F-j C\right\}<\phi_{v}^{j}(a) .
$$

The proof will then be finished by choosing $k$ so large that $\phi_{v}^{k}(a)<\eta$ and then taking $A=k C$.

We prove (6.8) by induction on $j$; the case $j=0$ is (6.5). From (2.3), we have in the notation of Lemma 6.1,

$$
\log \left|Z_{L+j+1}\right|=\log \left|q\left(\underline{x}, \underline{Z}_{L+j}\right)\right|+\max _{1 \leqq k \leqq d} \log \left|Z_{L+j}^{(k)}\right| .
$$

By the induction assumption (6.8) and the independence of the random variables $Z_{L+j}^{(k)}$,

$$
\text { Prob }\left\{\max _{1 \leqq k \leqq d} \log \left|Z_{L+j}^{(k)}\right| \leqq F-j C\right\}<\left(\phi_{v}^{j}(a)\right)^{d} .
$$

Since by (6.9), the inequality $\log \left|Z_{L+j+1}^{(k)}\right| \leqq F-(j+1) C$ can hold only if either $\max _{1 \leqq k \leqq d} \log \left|Z_{L+j}^{(k)}\right| \leqq F-j C$ or $\log |q(\underline{x}, \underline{z})|<-C$, (6.7) and (6.10) imply that

$$
\begin{aligned}
\operatorname{Prob}\left\{\log \left|Z_{L+j+1}^{(k)}\right| \leqq F-(j+1) C\right\} & <\left(\phi_{v}^{j}(a)\right)^{d}+v \\
& =\phi_{v}^{j+1}(a) .
\end{aligned}
$$

Now we can state and prove the main result of this section.

Theorem 6.4. If the free energy per site $\log \left|Z_{L}\right| / L$ is partially self averaging to the value $f$, then it is self averaging to the value $f$.

Proof. Given $\eta, \varepsilon>0$ we must show that for sufficiently large $L$,

$$
\text { Prob }\left\{\frac{\log \left|Z_{L}\right|}{L}<f-\varepsilon\right\}<\eta \text {, }
$$

and

$$
\operatorname{Prob}\left\{\frac{\log \left|Z_{L}\right|}{L}>f+\varepsilon\right\}<\eta \text {. }
$$

Now (6.13) follows directly from the fact that $\log \left|Z_{L}\right| / L$ is partially self averaging to the value $f$, and the latter also implies that there exist $a<1$ and $L_{0}>0$ such that for all $L>L_{0}$,

$$
\operatorname{Prob}\left\{\frac{\log \left|Z_{L}\right|}{L}<f-\frac{\varepsilon}{2}\right\}<a .
$$


But when (6.14) holds, Lemma 6.3 implies that for some $k$ and $A$ independent of $L$,

$$
\text { Prob }\left\{\frac{\log \left|Z_{L+k}\right|}{L+k}<f-\frac{\varepsilon}{2}-\frac{A+k(f-\varepsilon / 2)}{L+k}\right\}<\eta \text {. }
$$

We may assume that $L_{0}$ is so large that $(A+k f) / L_{0}<\varepsilon / 2$, so that (6.15) implies that (6.12) holds for $L>L_{0}+k$.

Theorem 5.2 and Theorem 6.4 immediately imply that the results presented in Sect. 2 hold in phases I and III:

Theorem 6.5. $\log \left|Z_{L}\right| / L$ is self averaging to $f_{\mathrm{I}}=\log (d|\langle x\rangle|)$ in phase $\mathrm{I}$, to $f_{\mathrm{III}}=\log \left(d\left\langle|x|^{2}\right\rangle\right) / 2$ in phase III, and to $f_{\mathrm{I}}=f_{\mathrm{III}}$ on the I-III phase boundary.

We finally show that the behavior of that $\log \left|Z_{L}\right| / L$ is closely related to that of $\log \left\langle\left\langle\left|Z_{L}^{2}\right|\right\rangle\right\rangle / 2 L$.

Theorem 6.6. $\log \left|Z_{L}\right| / L$ is self averaging to the value $f$ if and only if $\log \left\langle\left\langle\left|Z_{L}^{2}\right|\right\rangle / 2 L\right.$ is.

Proof. Let us write Prob $\left\{E \mid\left\{\left|x_{b}\right|\right\}\right\}$ for the probability, in the phase variables $s_{b}$, that some condition $E$ holds at the fixed values $\left\{\left|x_{b}\right|\right\}$ of the bond weight amplitudes. We now choose a number $a$ satisfying $0<a<1 / 24$ and apply Lemma 5.1, taking $Y=\left|Z_{L}^{2}\right|$ and using the bound $\left\langle\left\langle\left|Z_{L}\right|^{4}\right\rangle\right\rangle /\left\langle\left\langle\left. Z_{L}\right|^{2}\right\rangle\right\rangle^{2} \leqq 24$ of Theorem 4.2, to conclude: (a) for any $\varepsilon, \eta>0$ there is an $L_{0}$ such that for all $L>L_{0}$ and all $\left\{\left|x_{b}\right|\right\}$,

$$
\operatorname{Prob}\left\{\frac{\log \left|Z_{L}\right|}{L}>\frac{\log \left\langle\left\langle\left|Z_{L}\right|^{2}\right\rangle\right\rangle}{2 L}+\varepsilon \mid\left\{\left|x_{b}\right|\right\}\right\}<\eta ;
$$

and (b) for any $\varepsilon>0$ there is an $L_{0}$ such that for all $L>L_{0}$ and all $\left\{\left|x_{b}\right|\right\}$,

$$
\text { Prob }\left\{\frac{\log \left|Z_{L}\right|}{L}<\frac{\log \left\langle\left\langle\left|Z_{L}\right|^{2}\right\rangle\right.}{2 L}-\varepsilon \mid\left\{\left|x_{b}\right|\right\}\right\}<a .
$$

Now suppose that $\log \left\langle\left\langle\left|Z_{L}^{2}\right|\right\rangle\right\rangle / 2 L$ is self averaging to the value $f$. If we integrate (6.16) and (6.17) over the distribution of the bond weight amplitudes $\left\{\left|x_{b}\right|\right\}$ we conclude that for any $\varepsilon, \eta>0$,

$$
\operatorname{Prob}\left\{\frac{\log \left|Z_{L}\right|}{L}>\frac{\log \left\langle\left\langle\left|Z_{L}\right|^{2}\right\rangle\right\rangle}{2 L}+\varepsilon\right\}<\eta
$$

for all sufficiently large $L$; and that for any $\varepsilon>0$,

$$
\operatorname{Prob}\left\{\frac{\log \left|Z_{L}\right|}{L}<\frac{\log \left\langle\left\langle\left|Z_{L}\right|^{2}\right\rangle\right\rangle}{2 L}-\varepsilon\right\}<a
$$

for all sufficiently large $L$. These equations, together with the assumed self averaging property of $\log \left\langle\left\langle\left|Z_{L}^{2}\right|\right\rangle\right\rangle / 2 L$, imply immediately that $\log \left|Z_{L}\right| / L$ is partially self averaging to $f$. Thus by Theorem $6.4, \log \left|Z_{L}\right| / L$ is self averaging to $f$.

Now suppose conversely that $\log \left\langle\left\langle\left|Z_{L}\right|^{2}\right\rangle\right\rangle / 2 L$ is not self averaging to $f$. Then there exist $\varepsilon^{\prime}, \eta^{\prime}>0$ such that for any $L_{1}$ there exists an $L>L_{1}$ for which

$$
\operatorname{Prob}\left\{\left|\frac{\log \left\langle\left\langle\left|Z_{L}\right|^{2}\right\rangle\right\rangle}{2 L}-f\right|>\varepsilon^{\prime}\right\}>\eta^{\prime} .
$$


On the other hand, it follows directly from (6.16) and (6.17), taking $\eta=(1-a) / 2$ and $\varepsilon=\varepsilon^{\prime} / 2$, that there exists an $L_{0}$ and such that for all $L>L_{0}$ and all $\left\{\left|x_{b}\right|\right\}$,

$$
\operatorname{Prob}\left\{\left|\frac{\log \left|Z_{L}\right|}{L}-\frac{\log \left\langle\left\langle\left|Z_{L}\right|^{2}\right\rangle\right\rangle}{2 L}\right|<\frac{\varepsilon^{\prime}}{2} \mid\left\{\left|x_{b}\right|\right\}\right\}>\frac{1-a}{2} .
$$

Now certainly if $|\log | Z_{L} \mid / L-\log \left\langle\left\langle\left|Z_{L}^{2}\right|\right\rangle / 2 L\right|\left\langle\varepsilon^{\prime} / 2\right.$ and $\left|\log \left\langle\left\langle\left|Z_{L}^{2}\right|\right\rangle\right\rangle / 2 L-f\right|$ $>\varepsilon^{\prime}$, then $|\log | Z_{L}|/ L-f|>\varepsilon^{\prime} / 2$. Thus if we integrate (6.21) over those bond weight amplitudes for which $\left|\log \left\langle\left\langle\left|Z_{L}^{2}\right|\right\rangle\right\rangle / 2 L-f\right|>\varepsilon^{\prime}$, and use (6.20), we find that for any $L_{1} \geqq L_{0}$ there exists an $L>L_{1}$ such that

$$
\operatorname{Prob}\left\{\left|\frac{\log \left|Z_{L}\right|}{L}-f\right|>\frac{\varepsilon^{\prime}}{2}\right\}>\frac{(1-a) \eta^{\prime}}{2},
$$

so that $\log \left|Z_{L}\right| / L$ is not self averaging to $f$.

\section{The Free Energy in Phase II}

In this section we complete the proof of our main result by showing that in phase II, $\log \left|Z_{L}\right| / L$ is self averaging to the value $f_{\mathrm{II}}$. The main idea of the argument is that, by varying the distribution of the phase $s$ while keeping the distribution of the amplitude $|x|$ fixed, one can reduce the estimation of $\log \left|Z_{L}\right| / L$ to the corresponding problem in two simpler, well-understood cases: a system with positive bond weights in phase II ([2]) and a system in phase I arbitrarily close to the I-II phase boundary (see previous sections).

Let us first recall the known possible phases for the model we are considering in the case in which the bond weights are positive random variables $[2,10]$ :

Theorem 7.1. Suppose that the bond weight $x$ is positive. Then:

(a) If $\alpha_{\min }>1$ then $\log Z_{L} / L$ is self averaging to the value $f_{\mathrm{I}}=\log (d\langle x\rangle)$;

(b) If $\alpha_{\min } \leqq 1$ then $\log Z_{L} / L$ is self averaging to the value $f_{\mathrm{II}}=G\left(\alpha_{\min }\right)$.

It is easy to check that the conclusions of Theorem 7.1 are special cases of our general results summarized in Sect. 2. For when $x>0,(2.7)$ can never be satisfied, since it implies that $G(2)>G(1)$ and $\alpha_{\min }>2$, which is inconsistent with the fact that $\alpha_{\min }$ is the unique minimum of $G(\alpha)$, and hence the system cannot be in phase III. Moreover, the conditions (2.5) and (2.6) for phases I and II reduce respectively to $\alpha_{\min }>1$ and $\alpha_{\min }<1$, and the I-II phase boundary is the point $\alpha_{\min }=1$. Thus part (a) of the theorem follows from Theorem 6.5. For completeness we will sketch briefly a proof of part (b); for a full discussion see [2] (the conclusion there is in fact stronger, since it is proved that $\log Z_{L} / L$ converges to $f_{\mathrm{I}}$ or $f_{\mathrm{II}}$ in phase I or phase II almost surely, not just in probability).

Proof sketch, Theorem 7.1(b): We suppose that $\alpha_{\min } \leqq 1$ and consider the extension of the given model, which has (positive) bond weights $x_{b}$, to the family of models indexed by a positive parameter $T$, the temperature, with bond weights $x_{b}^{1 / T}$. For this family of models, the partition function $Z_{L}(T)$ is given by

$$
Z_{L}(T)=\sum_{W}\left(X_{W}\right)^{1 / T}
$$


and we define $g_{L}(T)$ by

$$
g_{L}(T)=T \log Z_{L}(T) / L
$$

This quantity is (up to a sign) the usual thermodynamic free energy per step. A direct calculation of the first two derivatives of $g_{L}(T)$ shows that $g_{L}(T)$, as a function of $T$, is increasing (thermodynamically, this corresponds to positivity of entropy) and convex (it is easy to see that $d^{2} g / d T^{2}$ is positive; thermodynamically, this corresponds to the positivity of the specific heat). To prove Theorem 7.1(b), we must calculate $g_{L}(1)$.

For this family of models, the convex function $G_{T}(\alpha)$ used in the criteria (2.5) $-(2.7)$ and the formulae $(2.10)-(2.12)$ is

$$
G_{T}(\alpha)=\frac{1}{\alpha} \log \left(d\left\langle\left(x^{1 / T}\right)^{\alpha}\right\rangle=\frac{1}{T} G(\alpha / T),\right.
$$

which takes its minimum (with respect to $\alpha$ ) at $T \alpha_{\min }$. Let $T_{0}=1 / \alpha_{\min }$. The behavior of the function $g_{L}(T)$ is shown schematically in Fig. 3, and may be summarized as follows.

(i) $T>T_{0}$ : For $T>T_{0}$ the model with parameter $T$ is in phase I, so that Theorem 7.1 (a) implies that $g_{L}(T)$ is self averaging to $T \log \left(d\left\langle x^{1 / T}\right\rangle\right)=G(1 / T)$.

(ii) $T \leqq T_{0}$ : Since (i) implies that for $T>T_{0}$ and $L$ large, $g_{L}(T) \simeq G(1 / T)$ with high probability, and since $G(1 / T)$ has a minimum at $T_{0}$, it follows that for $T \leqq T_{0}$, $g_{L}(T)<G\left(1 / T_{0}\right)+\varepsilon$ (because $g_{L}(T)$ is increasing) and $g_{L}(T)>G\left(1 / T_{0}\right)-\varepsilon$ $-\eta\left(T_{0}-T\right)$ (because $g_{L}$ is convex and therefore lies above any of its tangents). Here $\varepsilon$ and $\eta$ may be taken arbitrarily small, so that for $L$ sufficiently large, $g_{L}(T)$ for all $T \leqq T_{0}$ (and in particular $g_{L}(1)$, since $\alpha_{\min } \leqq 1$ ) is with high probability arbitrarily close to $G\left(1 / T_{0}\right)=G\left(\alpha_{\min }\right)=f_{\mathrm{II}}$.

We now turn to the main results of this section. Let us consider then a specific directed polymer model, with partition function $Z_{L}$, for which the distribution $\rho$ of the bond weights $x_{b}$ is such that the model is in phase II, that is, satisfies (2.6). It will be convenient to associate to the given model a family of models indexed by a parameter $t \in[0,1]$, the $t$-models; we will denote the partition function of the

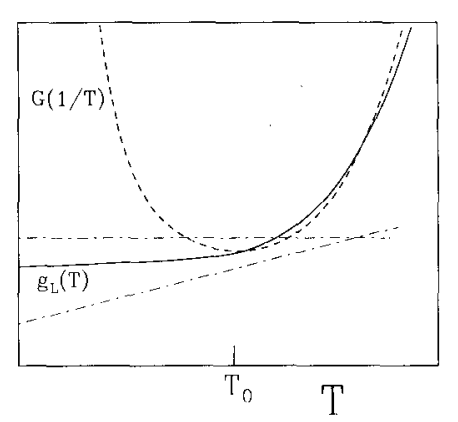

Fig. 3. Schematic representation of typical behavior of $g_{L}(T)=T \log Z_{L}(T) / L$ in the positive weight case, for $L$ large. For $T>T_{0}, g_{L}(T) \simeq G(1 / T)$ with high probability; for $T<T_{0}, g_{L}(T)$ is with high probability trapped between straight lines $G\left(1 / T_{0}\right)+\varepsilon$ and $G\left(1 / T_{0}\right)-\varepsilon-\eta\left(T_{0}-T\right)$, with $\varepsilon, \eta$ small 
$t$-model by $\tilde{Z}_{L}(t)$. By definition the $t$-model has amplitudes $\left|x_{b}\right|$ with the distribution of those in the original model and has phase variable $s_{b}(t)$ distributed according to

$$
s_{b}= \begin{cases}+1, & \text { with probability }(1+t) / 2, \\ -1, & \text { with probability }(1-t) / 2 .\end{cases}
$$

We introduce also the quantity $Y_{L}(t)$, defined for $0 \leqq t \leqq 1$ by

$$
Y_{L}(t)=\sum_{W_{1}} \sum_{W_{2}}\left|X_{W_{1}} X_{W_{2}}\right| t^{i\left(W_{1}, W_{2}\right)},
$$

where the sum is over pairs of walks of length $L$ and $l\left(W_{1}, W_{2}\right)$ is the number of bonds visited by exactly one of the two walks. It is clear that for all $t$,

$$
\left\langle\left\langle\left|\tilde{Z}_{L}(t)\right|^{2}\right\rangle\right\rangle=Y_{L}(t),
$$

and that

$$
\left\langle\left\langle\left|Z_{L}\right|^{2}\right\rangle\right\rangle=Y_{L}(|\langle s\rangle|) .
$$

It is also clear that $Y_{L}(t)$ is an increasing function of $t$ (this will be needed in the proof of Lemma 7.3). Moreover, we can determine the asymptotic behavior of $Y_{L}(t)$ for certain values of $t$ :

Lemma 7.2. (a) $\log Y_{L}(0) / 2 L$ is self averaging to the value $f_{\mathrm{II}}$;

(b1) if $\alpha_{\min } \leqq 1$, then $\log Y_{L}(1) / 2 L$ is self averaging to the value $f_{\mathrm{n}}$;

(b2) if $1<\alpha_{\min } \leqq 2$, then for any $\varepsilon<0$ there exists $t_{0}$, with $|\langle s\rangle|<t_{0}<1$, such that $\log Y_{L}\left(t_{0}\right) / 2 L$ is self averaging to a value $f_{0}$ satisfying $f_{0}<f_{\mathrm{II}}+\varepsilon$.

Proof. (a) When $t=0$ the only terms which contribute to (7.5) are those with $W_{1}=W_{2}$, so that

$$
Y_{L}(0)=\sum_{W}\left|X_{W}\right|^{2}=Z_{L}^{\prime},
$$

where $Z_{L}^{\prime}$ is the partition function for directed polymers on the tree with positive bond weight $x_{b}^{\prime}=\left|x_{b}\right|^{2}$. The phase of this latter system is determined by the function $G^{\prime}(\alpha)=2 G(2 \alpha)$, which has its minimum at $\alpha_{\min }^{\prime}=\alpha_{\min } / 2 \leqq 1$. Thus the primed system is in phase II or on the I-II phase boundary and by Theorem 7.1(b) the free energy $\log \left|Z_{L}^{\prime}\right| / L=\log Y_{L}(0) / L$ is self averaging to the value $f_{\mathrm{II}}^{\prime}=G^{\prime}\left(\alpha_{\min }^{\prime}\right)=2 G\left(\alpha_{\min }\right)=2 f_{\mathrm{II}}$.

(b) We wish to determine in what phase the $t$-model lies as $t$ is increased from $t=|\langle s\rangle|$ (relevant because of (7.6), (7.7)) to $t=1$. Now because the $t$-model has the same distribution of amplitudes as the original model, the function $G_{t}(\alpha)$ (see (2.4)), used to define the phases in the $t$-model, is in fact independent of $t$ and identical to $G(\alpha)$. From this observation, the criteria (2.5)-(2.7) of Sect. 2, and the hypothesis $\alpha_{\min } \leqq 2$ of the lemma, we see that:

(i) if $\alpha_{\min }<1$ then the $t$-model will be in phase II for all $t$;

(ii) if $\alpha_{\min }=1$ then the $t$-model will be in phase II for $|\langle s\rangle| \leqq t<1$ and on the I-II phase boundary for $t=1$;

(iii) if $\alpha_{\min }>1$ then the $t$-model will be in phase I whenever $t>t_{1}$, where $\log t_{1}=G\left(\alpha_{\min }\right)-\log (d\langle|x|\rangle)$, and in phase II whenever $t<t_{1}$; moreover, $t_{1} \geqq|\langle s\rangle|$ because the original model is in phase II and therefore the $t$-model with $t=|\langle s\rangle|$ is also in phase II. 
Suppose now that $\alpha_{\min } \leqq 1$, so that the $t=1$ model is in phase II or on I-II phase boundary. For $t=1$ the $t$-model has positive weights, and it follows from Theorem 7.1(b) that $\log \left|\tilde{Z}_{L}(1)\right| / L$ is self averaging to $f_{\mathrm{II}}$, and from Theorem 6.6 that $\log Y_{L}(1) / 2 L=\log \left\langle\left\langle\left|\tilde{Z}_{L}(1)\right|^{2}\right\rangle\right\rangle / 2 L$ is self averaging to the same value. This verifies part (b1) of Lemma 7.2.

On the other hand, if $\alpha_{\min }>1$, then (iii) above and Theorem $6.5 \mathrm{imply}$ that for $t>t_{1}, \log \left|\tilde{Z}_{L}(t)\right| / L$ is self averaging to the value $f_{\mathrm{I}}(t)=\log (d|\langle x(t)\rangle|)=$ $\log (d\langle|x|\rangle)+\log t$. As $\left.t\rangle t_{1}, f_{1}(t)\right\rangle G\left(\alpha_{\min }\right)=f_{\mathrm{II}}$, so that for any $\varepsilon>0$ we may find $t_{0}$ with $t_{1}<t_{0}<1$ for which $f_{0} \equiv f_{\mathrm{I}}\left(t_{0}\right)<f_{\mathrm{II}}+\varepsilon$. Again, Theorem 6.6 implies that $\log Y_{L}\left(t_{0}\right) / 2 L$ is also self averaging to $f_{0}$, completing the proof of part (b2) of Lemma 7.4 .

The next lemma is the critical result for our discussion of phase II.

Lemma 7.3. Suppose that $\alpha_{\min } \leqq 2$ and that $G(\alpha) \geqq \log (d|\langle x\rangle|)$ for all $\left.\alpha \in\right] 1,2[$. Then $\log \left\langle\left\langle\left|Z_{L}^{2}\right|\right\rangle\right\rangle / 2 L$ is self averaging to the value $f_{\mathrm{II}}$.

Proof. The result follows immediately from Lemma 7.2, since given $\varepsilon, \eta>0$ we have from $\left\langle\left\langle\left|Z_{L}\right|^{2}\right\rangle\right\rangle=Y_{L}(|\langle s\rangle|) \geqq Y_{L}(0)$ and Lemma 7.2(a) that for sufficiently large $L$,

$$
\operatorname{Prob}\left\{\frac{\log \left\langle\left\langle\left|Z_{L}\right|^{2}\right\rangle\right\rangle}{2 L}<f_{\mathrm{II}}-\varepsilon\right\}<\eta,
$$

while if Lemma 7.2(b1) applies we have from $\left\langle\left\langle\left|Z_{L}\right|^{2}\right\rangle\right\rangle=Y_{L}(|\langle s\rangle|) \leqq Y_{L}(1)$ that for sufficiently large $L$,

$$
\operatorname{Prob}\left\{\frac{\log \left\langle\left\langle\left|Z_{L}\right|^{2}\right\rangle\right\rangle}{2 L}>f_{\mathrm{II}}+\varepsilon\right\}<\eta,
$$

and if Lemma 7.2(b2) applies we have (with $t_{0}$ as in that lemma) from $\left\langle\left\langle\left|Z_{L}\right|^{2}\right\rangle=Y_{L}(\langle s\rangle) \leqq Y_{L}\left(t_{0}\right)\right.$ that for sufficiently large $L$,

$$
\operatorname{Prob}\left\{\frac{\log \left\langle\left\langle\left|Z_{L}\right|^{2}\right\rangle\right\rangle}{2 L}>f_{\mathrm{II}}+2 \varepsilon\right\}<\eta .
$$

The systems which satisfy the hypotheses of Lemma 7.3 are precisely those in phase II, on the I-II and II-III phase boundaries, and at the triple point. Thus, this lemma and Theorem 6.4 immediately imply the main result of this section:

Theorem 7.4. Suppose that the system is in phase II, on the I-II or II-III phase boundaries, or at the triple point. Then $\log \left|Z_{L}\right| / L$ is self averaging to the value $f_{\mathrm{II}}$.

Remark 7.5. In this paper, we have treated phase I and phase III in very similar ways, but for phase II have used the quite different arguments presented in this section. It is worth noticing that we could have limited the disucssion in Sects. 4-6 to phase I only, and then used the arguments of Sect. 7 to treat phases II and III in parallel. This is primarily because the free energy in both phase II and phase III does not depend on the distribution of the phase variable $s$, even through its mean $\langle s\rangle$. 


\section{Conclusion}

In this paper we have determined completely the asymptotic behavior of the partition function for the problem of directed polymers with complex random weights on the Cayley tree. In particular, we have shown that only the three phases determined by (2.5), (2.6) and (2.7) are possible, and that in these phases, $\log \left|Z_{L}\right| / L$ is self averaging to the value given by the expressions (2.10), (2.11) and (2.12), respectively. The main tools used to prove these results are an extension of a method based on the estimate of noninteger moments of the partition function $[2,11]$, which leads to the computation of the correct limiting value of the free energy per step and implies partial self averaging to it, and an argument suggested by the treatment of [6], which improves the probabilistic lower bound on the free energy per step and thus yields full self averaging.

These results were obtained only under the hypotheses $\mathrm{H} 1, \mathrm{H} 2$ and $\mathrm{H} 3$ on the distribution of the bond weights $x_{b}$. Each of these conditions is probably too restrictive. In particular, the hypothesis $\mathrm{H} 3$ that the moments $\left\langle|x|^{\alpha}\right\rangle$ are finite for all $\alpha>0$ can certainly be relaxed. It suffices to assume, for example, that these moments are finite in some interval $0<\alpha<\alpha_{0}$ and that the function $G(\alpha)$ of (2.4) attains a minimum at some point $\alpha_{\min }$ of this interval; the proofs of our results are essentially unchanged in this case. The hypothesis $\mathrm{H} 1$ that the distribution of $|x|$ is continuous was only used in the proof that the partial self averaging property implies full self averaging (Lemma 6.3 and Theorem 6.4). It seems likely that this result holds more generally: in the case of positive weights $\log Z_{L} / L$ has been shown to be self averaging $[2,6]$ with no similar restriction on the weight distribution.

The most serious restriction that we consider is $\mathrm{H} 2$, the independence of the phase $s$ and of the amplitude $|x|$. We used this condition repeatedly - in fact, whenever we averaged over phase variables in the estimates of Sect. 4 or in the discussion of phase II in Sect. 7. On the other hand, an example where the phase and amplitude are correlated has been studied, at least numerically (see example 1 of [5]), and the expression for $\log \left|Z_{L}\right| / L$ seems to remain valid. A method of proof which overcomes this restriction would be of interest because it would make possible the consideration of the case of real energies with a complex temperature (i.e., the case $x_{b}=\exp \left(-\beta \varepsilon_{b}\right)$, where the energies $\varepsilon_{b}$ are real random variables and the inverse temperature $\beta$ is complex).

Beyond the possibility of weakening these hypotheses, there are two natural ways of extending the results of the present work to other models. First, one could try to use the same approach to study the Random Energy Models, REM [7]. In these models the partition function $Z_{L}$ may be written as in (2.2),

$$
Z_{L}=\sum_{1 \leqq W \leqq d^{L}}\left|X_{W}\right| S_{W},
$$

where as before the amplitude $\left|X_{W}\right|$ and the phase $S_{W}$ are products of random weights:

$$
\left|X_{W}\right|=\prod_{1 \leqq b \leqq L}\left|x_{W, b}\right| \quad \text { and } \quad S_{W}=\prod_{1 \leqq b \leqq L} s_{W, b},
$$

but where, in contrast with the directed polymer problem, the variables $\left|x_{W, b}\right|$ and $s_{W, b}$ associated to distinct walks are independent. Most of what is said in the present paper remains valid in this case (some of the calculations of Sect. 4 can even be greatly simplified). To reproduce our results fully, however, would require that 
the proof of Theorem 6.4, which is based on the recursion relation (2.3) for $Z_{L}$, be modified in order to show that in the REM, too, partial self averaging implies full self averaging. In the REM, as in the directed polymer model, we do not know at the moment how to generalize our approach to situations where the phase $S_{W}$ and the amplitude $X_{W}$ of a given walk $W$ are correlated, although in the case of the REM, too, recent work $[9,23]$ indicates the validity of the results in cases where the hypothesis $\mathrm{H} 2$ is not fulfilled. A full discussion of the REM, including the case of correlated $X_{W}$ and $S_{W}$, would be useful to confirm some recent predictions on the location of the zeroes of the partition function of the REM in the complex plane of the temperature $[9,23]$.

The other possible generalization of our work is to the directed polymer problem with random complex weights in finite dimension $d$. In that case, known methods for the positive weight case $([1,4,11,16])$ suffice to establish the existence of a phase $\mathrm{I}$ in dimension $d>3$, in which $\log \left|Z_{L}\right| / L$ is still self averaging to $f_{\mathrm{I}}$. This is because $([4,11,16])$ one may obtain a bound for the ratio $\left\langle\left|Z_{L}\right|^{2}\right\rangle /\left|\left\langle Z_{L}\right\rangle\right|^{2}$ as long as the probability that two directed walks never meet is nonzero (in the model in which all bonds have weight 1 ). The calculation of the boundary of phase I, and of $\log \left|Z_{L}\right| / L$ outside this phase, seems to be a much more difficult problem.

At the moment we do not know whether in high dimension the phase diagram is similar to that of the tree problem, with only 3 phases, or if there exist two additional phases (phases IV and V) as predicted by a recent replica calculation [12]. Some progress might be made through attempting to give bounds on moment ratios (of integer or noninteger moments) in parts of the phase plane for which the two theories disagree. One expects, however, that this method will at best give bounds on the positions of the phase boundaries and of the asymptotic value of $\log \left|Z_{L}\right| / L$, rather than exact expressions as given in the tree problem. If this is the case, there will remain some regions in the phase diagrams where the approach will be insufficient and where it would be hard to exclude the existence of new phases. Another possible way of checking the replica approach would be to apply the same replica scheme used in [12] directly to the tree problem, to see whether it still predicts the existence of the two extra phases IV and V. An affirmative answer would show that the method is incorrect at least for the tree and would suggest that other replica schemes be investigated.

Acknowledgements. M.R.E. thanks the Royal Society for financial support during this work. E.R.S. thanks the Service de Physique Theorique, Saclay, for hospitality while much of this work was done, and thanks T. Spencer for hospitality at the Institute for Advanced Study.

\section{References}

1. Bolthausen, E: A note on the diffusion of directed polymers in a random environment. Commun. Math. Phys. 123, 529-534 (1989)

2. Buffet, E., Patrick, A., Pulé, J.V.: Directed Polymers on Trees: a Martingale Approach. J. Phys. A26, 1823-1834 (1993)

3. Chauvin, B., Rouault, A.: KPP equation and supercritical branching Brownian motion in the subcritical speed area. Application to spatial trees. Prob. Th. and Relat. Fields 80, 299-314 (1988)

4. Cook, J., Derrida, B.: Polymers on disordered hierarchical lattices: A nonlinear combination of random variables. J. Stat. Phys. 57, 89-139 (1989)

5. Cook, J., Derrida, B.: Lyapounov exponents of large, sparse random matrices and the problem of directed polymers with complex random weights. J. Stat. Phys. 61, 961-986 (1990) 
6. Dekking, F.M.: A nonlinear evolution with travelling waves. In: Luck, J.M., Moussa, P., Waldschmidt, M. (eds.), Number Theory and Physics. Proceedings in Physics 47, pp. 204208. Berlin, Heidelberg, New York: Springer 1990

7. Derrida, B.: Random energy model: Limit of a family of disordered models. Phys. Rev. Lett. 45, 79-82 (1980)

8. Derrida, B.: Directed polymers in a random medium. Physica A163, 71-84 (1990)

9. Derrida, B.: The zeroes of the partition function of the random energy model. Physica A177, 31-37 (1991)

10. Derrida, B., Spohn, H.: Polymers on disordered trees, spin glasses and travelling waves. J. Stat. Phys. 51, 817-840 (1988)

11. Evans, M.R., Derrida, B.: Improved bounds for the transition temperature of directed polymers in a finite dimensional random medium. J. Stat. Phys. 69, 427-437(1992)

12. Goldschmidt, Y.Y., Blum, T.: Directed walks with complex random weights: Phase diagram and replica symmetry breaking. J. Phys. I (France) 2, 1607-1619 (1992)

13. Halpin-Healy, T.: Diverse manifolds in random media. Phys. Rev. Lett. 62, 442-445 (1989)

14. Huse, D.A., Henley, C.L.: Pinning and roughening of domain walls in Ising systems due to random impurities. Phys. Rev. Lett. 54, 2708-2711 (1985)

15. Huse, D.A., Henley, C.L., Fisher, D.S.: Response to a comment. Phys. Rev. Lett. 55, 29242924 (1985)

16. Imbrie, J.Z., Spencer, T.J.: Diffusion of directed polymers in a random environment. J. Stat. Phys. 52, 609-626 (1988)

17. Kardar, M.: Domain walls subject to quenched impurities. J. Appl. Phys. 61, 3601-3604 (1987)

18. Kardar, M., Parisi, G., Zhang, Y.-C.: Dynamic scaling of growing interfaces. Phys. Rev. Lett. 56, 889-892 (1986)

19. Kardar, M., Zhang, Y.-C.: Scaling of directed polymers in a random medium. Phys. Rev. Lett. 58, 2087-2090 (1987)

20. Krug, J., Spohn, H.: Kinetic Roughening of Growing Surfaces. In: Godrèche, C. (ed.), Solids far from Equilibrium. Cambridge, UK: Cambridge University Press 1991

21. Medina, E., Kardar, M., Shapir, Y., Wang, X.R.: Interference of directed paths in disordered systems. Phys. Rev. Lett. 62, 941-944 (1989)

22. Mezard, M., Parisi, G.: Replica field theory for random manifolds. J. Physique I1, 809-836 (1991)

23. Moukarzel, C., Parga, N.: Numerical complex zeros of the random energy model. Physica A177, 24-30 (1991)

24. Nguyen, V.L., Spivak, B.Z., Shklovskiǐ, B.I.: Tunnel hopping in disordered systems. JETP Sov. Phys. 62, 1021-1029 (1985)

25. Parisi, G.: On the replica approach to random directed polymers in two dimension. J. Physique 51, 1595-1606 (1990)

26. Shapir, Y., Wang, X.R.: Absence of h/e periodicity of the Aharonov-Bohm oscillations in square metallic lattices. Europhys. Lett. 4, 1165-1170 (1987)

27. Zhang, Y.C.: Ground state instability of a random system. Phys. Rev. Lett. 59, 2125-2128 (1987)

28. Zhang, Y.C.: Directed polymers with complex amplitudes. Phys. Rev. Lett. 62, 979-979 (1989)

29. Zhang, Y.C.: Directed polymers with complex amplitudes. Europhys. Lett. 9, 113-118 (1989) 